\title{
Substitutions of red meat, poultry and fish and risk of myocardial infarction
}

\author{
Anne M. L. Würtz ${ }^{1 *}$, Mette D. Hansen ${ }^{1}$, Anne Tjønneland ${ }^{2}$, Eric B. Rimm ${ }^{3,4}$, Erik B. Schmidt ${ }^{5}$, \\ Kim Overvad $^{1,5}$ and Marianne U. Jakobsen ${ }^{1}$ \\ ${ }^{1}$ Department of Public Health, Section for Epidemiology, Aarhus University, DK-800O Aarbus C, Denmark \\ ${ }^{2}$ Danish Cancer Society Research Center, DK-2100 Copenhagen Ø, Denmark \\ ${ }^{3}$ Department of Medicine, Channing Division of Network Medicine, Brigham and Women's Hospital, Harvard Medical School, \\ Boston, MA 02115, USA \\ ${ }^{4}$ Departments of Epidemiology and Nutrition, Harvard T. H. Chan School of Public Health, Boston, MA O2115, USA \\ ${ }^{5}$ Department of Cardiology, Center for Cardiovascular Research, Aalborg University Hospital, DK-9000 Aalborg, Denmark
}

(Submitted 2 June 2015 - Final revision received 15 December 2015 - Accepted 22 January 2016 - First published online 7 March 2016)

\section{Abstract}

Red meat has been suggested to be adversely associated with risk of myocardial infarction (MI), but previous studies have rarely taken replacement foods into consideration. We aimed to investigate optimal substitutions between and within the food groups of red meat, poultry and fish for MI prevention. We followed up 55171 women and men aged 50-64 years with no known history of MI at recruitment. Diet was assessed by a validated 192-item FFQ at baseline. Adjusted Cox proportional hazard models were used to calculate hazard ratios (HR) and $95 \%$ CI for specified food substitutions of $150 \mathrm{~g}$ /week. During a median follow-up time of 13.6 years, we identified 656 female and 1694 male cases. Among women, the HR for replacing red meat with fatty fish was 0.76 (95\% CI 0.64, 0.89), whereas the HR for replacing red meat with lean fish was 1.00 (95\% CI 0.89 1.14). Similarly, replacing poultry with fatty but not lean fish was inversely associated with MI: the HR was 0.81 (95\% CI 0.67, 0.98) for fatty fish and was 1.08 (95\% CI 0.92, 1.27) for lean fish. The HR for replacing lean with fatty fish was 0.75 (95\% CI $0 \cdot 60,0 \cdot 94)$. Replacing processed with unprocessed red meat was not associated with MI. Among men, a similar pattern was found, although the associations were not statistically significant. This study suggests that replacing red meat, poultry or lean fish with fatty fish is associated with a lower risk of MI.

Key words: Myocardial infarction: Red meat: Poultry: Fish: Cohort studies: Substitution models

Myocardial infarction (MI) remains a leading cause of morbidity and mortality in Western countries, and diet is an important modifiable risk factor ${ }^{(1)}$. When investigating the intake of foods in relation to risk of $\mathrm{MI}$, it is important to consider not only a specific food item but also the foods that may be replaced by or replace this food ${ }^{(2)}$. This is essential because the health impact per se of the specific food item cannot be isolated from that of other foods that it replaces.

Among foods, red meat is of particular interest because of its contents of SFA, dietary cholesterol and haem Fe, which have all been adversely associated with MI risk ${ }^{(3-5)}$. However, the existing literature on the association between intake of red meat and risk of $\mathrm{MI}$ is inconsistent ${ }^{(6)}$, possibly because previous studies have not specified the replacement food in the statistical models. The most obvious alternatives to red meat are poultry and fish. As poultry and fish contain less SFA, dietary cholesterol and haem Fe than red meat, and because fatty fish is a main source of $n-3$ PUFA, replacement of red meat by these foods is expected to be beneficial in MI prevention ${ }^{(2,7)}$.
In addition, the various cuts of red meat and different subtypes of fish may be differentially associated with MI incidence as a consequence of their varying amounts of both health-promoting and potentially harmful constituents ${ }^{(8)}$. Meat processing should also be taken into consideration, as a higher intake of processed red meat has been more consistently associated with a higher risk of MI compared with unprocessed red meat ${ }^{(6)}$.

We conducted a follow-up study to investigate optimal substitutions between and within the following food groups for MI prevention: red meat, poultry and fish.

\section{Methods \\ Study design and population}

The Diet, Cancer and Health study was initiated in the period between December 1993 and May 1997 by inviting 79729 women and 80996 men of whom 57053 consented to participate. All of them were 50-64-year-old citizens of the

Abbreviations: HR, hazard ratio; MI, myocardial infarction.

* Corresponding author: A. M. L. Würtz, fax +458613 1580, email amlw@ph.au.dk 
greater Copenhagen and Aarhus areas, born in Denmark and not previously registered in the Danish Cancer Registry. The protocols for the Diet, Cancer and Health study and for the present substudy were approved by the regional ethics committees on human studies in Aarhus and Copenhagen and by the Danish Data Protection Agency. A detailed description of the Diet, Cancer and Health cohort has been given elsewhere ${ }^{(9)}$.

\section{Exposure assessment}

Habitual diet over the past 12 months was assessed by a 192-item semi-quantitative FFQ filled in by the study participants before their visit to one of the two study centres in Copenhagen and Aarhus. Descriptions of the development and validation of the FFQ have been given elsewhere ${ }^{(10,11)}$. The participants reported the average dietary consumption of foods and beverages in twelve response categories ranging from 'never' to 'eight times or more per day'. The FFQ was processed by an optical scan to check for reading errors and missing information. Any uncertainty was clarified with the study participant, and no missing values were accepted. The daily intake of different foods was calculated in FoodCalc ${ }^{(12)}$, using sexspecific portion sizes ${ }^{(13,14)}$. In total, sixty-three questions covered the intake of meat and fish items and dishes, also providing detailed information on intakes of meat and fish subtypes. For the present study, meat was subdivided into red meat, consisting of both unprocessed and processed red meat, and poultry. Unprocessed red meat included fresh and minced beef, veal, pork and lamb. This group was further subdivided into lean $(\leq 10 \mathrm{~g})$ $100 \mathrm{~g})$ and fatty $(>10 \mathrm{~g} / 100 \mathrm{~g})$ unprocessed red meat. Processed red meat consisted of red meat items that had undergone processing such as smoking, salting or curing. This included various kinds of sausages, salami, smoked or cooked ham, other cold cuts, bacon and liver pate. Poultry included chicken and turkey. Total fish included all unprocessed and processed fish as well as shellfish and was further subdivided into two groups according to the content of $n$-3 PUFA: fish with low contents of $n-3$ PUFA $(\leq 1 \mathrm{~g} / 100 \mathrm{~g})$ included lean white fish, canned tuna and sardines, shellfish and cod roe, and fish with high contents of $n-3$ PUFA $(>1 \mathrm{~g} / 100 \mathrm{~g})$ included darker fish such as salmon, trout and herring, fresh and canned mackerel, and lumpfish roe.

\section{Covariates}

At enrolment, the participants filled in a lifestyle questionnaire containing questions on health status, social factors, lifestyle habits and reproductive factors. The participants provided information on smoking habits, physical activity, length of schooling, medical history and known hypertension, hypercholesterolaemia and diabetes mellitus; women provided information on menopausal status and use of hormone replacement therapy (HRT) as well. Similar to the FFQ, the lifestyle questionnaire was checked for reading errors and missing information to be clarified with each study participant, although a few missing values were accepted in the lifestyle questionnaire.

At the visit to the study clinic, height, weight and waist circumference were recorded by trained health professionals.
Information on alcohol consumption, intake of other foods and total energy intake was obtained from the FFQ.

\section{Case ascertainment}

The outcome measures were incident non-fatal and fatal MI. Participants registered with a first-time discharge diagnosis of MI or cardiac arrest believed to be caused by an MI (International Classification of Diseases, 8th revision, codes 410410.99 and $427 \cdot 27$, and International Classification of Diseases, 10th revision, codes I21.0-I21.9 and I46.0-I46.9) were identified in the Danish National Patient Register and in the Danish Cause of Death Register using the unique ten-digit civil registration number assigned to all Danish citizens by the Central Population Register. From baseline until 2003, the medical records of potential cases were reviewed, and the cases were validated according to MI criteria set by the American Heart Association and the European Society of Cardiology for use in epidemiology $^{(15)}$. From 1 January 2004 until 31 December 2009, participants with a MI diagnosis from a hospital ward were accepted as cases without further validation, as the positive predictive value of these register diagnoses from hospital wards is higher than $92 \%{ }^{(16)}$. Other potential cases were validated by review of diagnosis and procedure codes in the National Patient Register and the Cause of Death Register. Information on vital status and emigration was obtained by linkage with the Danish Civil Registration System.

\section{Exclusions}

Participants diagnosed with cancer but not registered in the Danish Cancer Registry before baseline were subsequently excluded (owing to the original focus area of the study). Furthermore, participants with a diagnosis of MI or cardiac arrest before enrolment and participants who did not fill out the lifestyle questionnaire or had missing information on potential confounders were excluded.

\section{Statistical methods}

The study participants were followed up from the date of study entry until the date of MI diagnosis, emigration, death, loss to follow-up or 31 December 2009, whichever occurred first. The associations between meat and fish substitutions and incidence of MI were investigated by Cox proportional hazards models, in which hazard ratios (HR) and corresponding 95\% CI were calculated. Age was included as the underlying timescale. The analyses were carried out among all participants in sex-stratified analyses and separately for women and men. The observation time was calculated as the time between study entry and end of follow-up for each participant.

Baseline characteristics of the study participants were summarised using medians and $80 \%$ central ranges for continuous variables and proportions for categorical variables. Intakes of meat and fish were energy adjusted, using the residual method ${ }^{(17)}$. The residual energy-adjusted variables were only used for descriptive purposes. 
We investigated differences in intake of $150 \mathrm{~g} /$ week, which reflects a usual serving size of red meat. The substitution aspect was introduced into the statistical models by inclusion of a variable made up by the sum of the food groups red meat, poultry and fish in addition to each of these food groups separately, except for the food group to be substituted. Accordingly, the HR was interpreted as the estimated risk of MI per $150 \mathrm{~g} /$ week higher intake of one of the specific food groups and a concomitant $150 \mathrm{~g} /$ week lower intake of the food group excluded from the model. For sensitivity analyses, we also investigated substitutions in $\mathrm{kJ} /$ week.

All associations were investigated with adjustment for total energy (kJ/week; continuous) (model 1a) and further adjustment for the potential confounders: BMI ( $\mathrm{kg} / \mathrm{m}^{2} ;$ continuous), waist circumference ( $\mathrm{cm}$; continuous), alcohol abstinence (yes, no), alcohol intake ( $\mathrm{g} / \mathrm{d}$; continuous), smoking status and amount (never, former, current $<15 \mathrm{~g}$ tobacco/d, current $15-25 \mathrm{~g}$ tobacco/d and current $>25 \mathrm{~g}$ tobacco/d), leisure-time physical activities (dichotomised; < or $\geq 3.5 \mathrm{~h} /$ week) and duration of schooling $(<8,8-10,>10$ years) as a measure of socio-economic status, in addition to menopausal status (premenopausal, postmenopausal, unknown) and use of HRT (never, former, current) among women (model 1b). The continuous variables BMI, waist circumference and alcohol intake were entered using restricted cubic splines with three knots. We further adjusted for the following food and beverage groups: vegetables, fruits, sweets, soft drinks, lean dairy products, fatty dairy products, fatty potatoes, refined cereals, whole-grain cereals and nuts (all g/week; continuous) (model 2). To investigate possible effect modification, we initially carried out analyses separately for participants with and without a baseline history of hypertension (yes, no, do not know), hypercholesterolaemia (yes, no, do not know) and/or diabetes mellitus (yes, no, do not know). No substantial differences were found, and we adjusted for these conditions in an additional model (results not shown).

Possible differences in the underlying dietary patterns related to total intake of red meat and fish were investigated using radar charts. Among women and men in the lowest and highest quintiles of meat or fish intake, we compared median intake of other foods and beverages with the median intake among the entire group, using the latter as denominator to calculate percentage differences in intake. For the radar charts, the foods were investigated in $\mathrm{g} /$ week and were all energy adjusted using the residual method ${ }^{(17)}$. All statistical analyses were performed in Stata 14 (StataCorp LP).

\section{Results}

A total of 29875 women and 27178 men agreed to participate, corresponding to $35 \%$ of those invited. Of these 57053 participants, 567 with a cancer diagnosis before baseline not initially registered and 900 with a baseline diagnosis of previous MI were excluded. Furthermore, forty-two participants missing a full baseline examination and 373 participants with missing information on potential confounders were excluded. These exclusions left 29142 female and 26029 male participants in the present study.

During a median follow-up of 13.6 (5th-95th percentile 8.6-15.1) years for women and 13.5 (5th-95th percentile 5.0-15.1) years for men, 656 female and 1694 male cases were identified. Baseline characteristics of the cohort members and cases are presented in Table 1. Among both women and men, cases were older, had a slightly higher BMI, a larger waist circumference, were more likely to be alcohol abstainers and

Table 1. Baseline characteristics of cohort and cases in the Diet, Cancer and Health study (Medians and 10th-90th percentiles or proportions)

\begin{tabular}{|c|c|c|c|c|c|c|c|c|}
\hline \multirow[b]{3}{*}{ Characteristics } & \multicolumn{4}{|c|}{ Women } & \multicolumn{4}{|c|}{ Men } \\
\hline & \multicolumn{2}{|c|}{ Cohort (n 29142) } & \multicolumn{2}{|c|}{ Cases $(n 656)$} & \multicolumn{2}{|c|}{ Cohort (n 26 029) } & \multicolumn{2}{|c|}{ Cases $(n$ 1694) } \\
\hline & Median & $\begin{array}{l}\text { 10th-90th } \\
\text { percentile }\end{array}$ & Median & $\begin{array}{l}\text { 10th-90th } \\
\text { percentile }\end{array}$ & Median & $\begin{array}{l}\text { 10th-90th } \\
\text { percentile }\end{array}$ & Median & $\begin{array}{l}\text { 10th-90th } \\
\text { percentile }\end{array}$ \\
\hline \multicolumn{9}{|l|}{ Physiological and anthropometric } \\
\hline Age (years) & 56 & $51-63$ & 59 & $52-64$ & 55 & $51-63$ & 57 & $51-63$ \\
\hline $\operatorname{BMI}\left(\mathrm{kg} / \mathrm{m}^{2}\right)$ & 25 & $21-31$ & 26 & $21-33$ & 26 & $22-31$ & 27 & 23-32 \\
\hline Waist circumference $(\mathrm{cm})$ & 80 & $69-97$ & 84 & $70-103$ & 95 & 84-109 & 97 & $86-112$ \\
\hline Postmenopausal $(\%)^{\star}$ & \multicolumn{2}{|c|}{59} & \multicolumn{2}{|c|}{70} & \multicolumn{2}{|r|}{ (1) } & \multicolumn{2}{|r|}{ 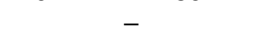 } \\
\hline \multicolumn{9}{|l|}{ Behavioural } \\
\hline Alcohol abstainer (\%) & \multicolumn{2}{|c|}{3} & \multicolumn{2}{|c|}{5} & \multicolumn{2}{|c|}{2} & \multicolumn{2}{|c|}{3} \\
\hline Alcohol intake $(g / d) \dagger$ & 10 & $1-35$ & 7 & $1-32$ & 20 & $5-63$ & 19 & $3-64$ \\
\hline Current smoker (\%) & \multicolumn{2}{|c|}{33} & \multicolumn{2}{|c|}{54} & \multicolumn{2}{|c|}{40} & \multicolumn{2}{|c|}{53} \\
\hline$<3.5 \mathrm{~h} /$ week physical activity (\%) & \multirow{2}{*}{\multicolumn{2}{|c|}{$\begin{array}{l}59 \\
31\end{array}$}} & \multicolumn{2}{|c|}{67} & \multicolumn{2}{|c|}{62} & \multicolumn{2}{|c|}{67} \\
\hline$<8$ years of education $(\%)$ & & & \multirow{2}{*}{\multicolumn{2}{|c|}{$\begin{array}{l}44 \\
30\end{array}$}} & \multirow{2}{*}{\multicolumn{2}{|c|}{34}} & \multicolumn{2}{|c|}{43} \\
\hline \multirow{2}{*}{\multicolumn{9}{|c|}{ Clinical }} \\
\hline & & & & & & & & \\
\hline Diabetes mellitus (\%) & \multicolumn{2}{|c|}{2} & \multicolumn{2}{|c|}{4} & \multicolumn{2}{|c|}{3} & \multicolumn{2}{|c|}{6} \\
\hline Hypertension (\%) & \multicolumn{2}{|c|}{17} & & & & & & \\
\hline Hypercholesterolaemia (\%) & & ; & & & & & & \\
\hline
\end{tabular}

* Among women

† Among users. 
Table 2. Energy-adjusted intakes of meat, poultry and fish among women and men in the Diet, Cancer and Health study (Medians and 10th-90th percentiles)

\begin{tabular}{|c|c|c|c|c|}
\hline \multirow[b]{2}{*}{ Energy-adjusted dietary intake (g/week) } & \multicolumn{2}{|c|}{ Women (n 29142$)$} & \multicolumn{2}{|c|}{ Men $(n 26029)$} \\
\hline & Median & 10th-90th percentile & Median & 10th-90th percentile \\
\hline Total red meat & 582 & $323-883$ & 973 & $604-1420$ \\
\hline Unprocessed red meat & 433 & $237-675$ & 684 & $401-1060$ \\
\hline Low fat $(\leq 10 \%)$ & 138 & $62-252$ & 227 & $105-414$ \\
\hline High fat $(>10 \%)$ & 285 & $156-449$ & 446 & $261-683$ \\
\hline Processed red meat & 133 & $46-271$ & 260 & $113-481$ \\
\hline Poultry & 115 & $37-270$ & 142 & $48-310$ \\
\hline Fish & 258 & $115-467$ & 303 & $133-550$ \\
\hline Low $n$-3 PUFA ( $\leq 1 \%)$ & 153 & $69-287$ & 178 & $75-339$ \\
\hline High $n-3$ PUFA $(>1 \%)$ & 90 & $27-215$ & 110 & $34-257$ \\
\hline
\end{tabular}

had lower alcohol intakes compared with the cohort. Furthermore, cases were more likely to be smokers, had lower levels of physical activity and had lesser education compared with the cohort. Among women, cases were more likely to be postmenopausal compared with cohort members, but no differences were observed regarding the use of HRT. Table 2 presents the energy-adjusted intakes of meat and fish among women and men.

Table 3 shows the HR and 95\% CI per $150 \mathrm{~g} /$ week higher intake of a specific type of meat, poultry or fish and a concomitant $150 \mathrm{~g}$ /week lower intake of another specified type of meat, poultry or fish among all participants and separately for women and men. Among women, the results from the minimally adjusted models suggested that replacing red meat with poultry or fish was associated with a lower risk of MI (model 1a). However, after adjustment for MI risk factors, risk estimates were weakened and for poultry they were no longer statistically significant (model 1b). The HR were 0.93 (95\% CI $0.84,1.04$ ) for substitution of poultry for red meat and 0.89 (95\% CI 0.82, 0.98) for substitution of fish for red meat. Between subgroups of foods, replacing unprocessed red meat with fish was associated with a lower risk of MI, HR 0.87 (95\% CI $0.79,0.97$ ), but no association was found when replacing processed red meat with fish, HR 0.93 (95\% CI 0.82, 1.06). Replacing red meat with fatty fish was associated with a lower risk of MI, HR 0.76 (95\% CI 0.64, 0.89), but no association was found when replacing red meat with lean fish, HR 1.00 (95\% CI $0.89,1.14)$. Similarly, replacing poultry with fatty fish was associated with a lower risk of MI, HR $0 \cdot 81$ (95\% CI 0.67, 0.98), whereas no association was found with lean fish, HR 1.08 (95\% CI $0.92,1.27)$. Generally, adjustment for other foods and food groups associated with MI attenuated the results only slightly (model 2). No major changes were observed with further adjustment for baseline history of hypertension, hypercholesterolaemia and diabetes mellitus (results not shown). Within food groups, the HR for replacing lean fish with fatty fish was 0.75 (95\% CI 0.60, 0.94). Replacing processed red meat with unprocessed red meat was not associated with risk of MI, HR 1.07 (95\% CI $0.93,1.22$ ). Among men, no statistically significant associations were observed, but the findings indicated a pattern similar to that of women.

Fig. 1 shows the underlying dietary patterns, represented by fourteen food and beverage groups, associated with low and high intakes of red meat and low and high intakes of fish among women and men separately. Panel a indicates that women in the lowest quintile of red meat intake consumed more fruits, vegetables, whole-grain cereals and lean and fatty dairy products and consumed less poultry, potatoes, fatty potatoes, soft drinks and alcohol than women in the highest quintile of red meat intake. A similar pattern was found among men. Panel $b$ indicates that women in the highest quintile of fish intake consumed more poultry, fruits, vegetables and alcohol and less sugar, sweets and snacks than women in the lowest quintile of fish intake. A similar pattern was found among men.

\section{Discussion}

In this follow-up study, we found an inverse association with MI among women when replacing red meat, poultry or lean fish with fatty fish. We found no association with MI when processed red meat was replaced with unprocessed red meat. A similar pattern, but no statistically significant associations, was found among men.

We compared the habitual diet of the study participants rather than investigating individual dietary changes over time. We compared participants who had an identical total energy intake and an identical total intake of red meat, poultry and fish, but for whom the intake of each of the three food groups differed. Controlling for the total amount of red meat, poultry and fish and at the same time including two of the three food groups in the statistical models allowed us to specify the substitutions between these three food groups. This is different from most other studies as meat and fish consumption has typically been investigated without considering replacement of food items. The substitution aspect is important, because individuals must alter their intake of specific foods primarily by changing their dietary composition rather than by changing their total energy intake, unless physical activity or body weight is changed considerably ${ }^{(18)}$. As foods are beneficial, neutral or harmful in relation to MI development, the association between red meat and MI depends on the replacement food.

The results presented in this study are all based on substitutions of food intakes of $150 \mathrm{~g} /$ week, corresponding to one serving size, which is easily translated into dietary guidelines for the general population. We also investigated substitutions of equal amounts 
Table 3. Myocardial infarction associated with substitutions of $150 \mathrm{~g} /$ week between and within the food groups of red meat, poultry and fish among women and men separately and combined in the Diet, Cancer and Health study

(Hazard ratios (HR) and $95 \%$ confidence intervals)

\begin{tabular}{|c|c|c|c|c|c|c|c|c|c|c|c|c|c|c|c|c|c|c|}
\hline \multirow[b]{3}{*}{ HR per $150 \mathrm{~g} /$ week } & \multicolumn{6}{|c|}{ All $(n 55171 / 2350)^{*}$} & \multicolumn{6}{|c|}{ Women (n 29 142/656) } & \multicolumn{6}{|c|}{ Men ( $n 26$ 029/1694) } \\
\hline & \multicolumn{2}{|c|}{ Model 1a† } & \multicolumn{2}{|c|}{ Model 1b $\ddagger$} & \multicolumn{2}{|c|}{ Model $2 \S$} & \multicolumn{2}{|c|}{ Model 1a† } & \multicolumn{2}{|c|}{ Model 1b $\ddagger$} & \multicolumn{2}{|c|}{ Model $2 \S$} & \multicolumn{2}{|c|}{ Model 1a† } & \multicolumn{2}{|c|}{ Model 1b $\ddagger$} & \multicolumn{2}{|c|}{ Model $2 \S$} \\
\hline & $\mathrm{HR}$ & $95 \% \mathrm{Cl}$ & HR & $95 \% \mathrm{Cl}$ & $\mathrm{HR}$ & $95 \% \mathrm{Cl}$ & $\mathrm{HR}$ & $95 \% \mathrm{Cl}$ & $\mathrm{HR}$ & $95 \% \mathrm{Cl}$ & $\mathrm{HR}$ & $95 \% \mathrm{Cl}$ & $\mathrm{HR}$ & $95 \% \mathrm{Cl}$ & HR & $95 \% \mathrm{Cl}$ & $\mathrm{HR}$ & $95 \% \mathrm{Cl}$ \\
\hline \multicolumn{19}{|c|}{ Substitutions between main food groups } \\
\hline Poultry for red meat & 0.95 & $0.90,0.99$ & 1.00 & $0.95,1.05$ & 1.00 & $0.95,1.05$ & 0.87 & $0.78,0.97$ & 0.93 & $0.84,1.04$ & 0.95 & $0.85,1.06$ & 0.96 & $0.91,1.02$ & 1.02 & $0.96,1.07$ & 1.01 & $0.96,1.07$ \\
\hline Poultry for unprocessed red meat & 0.98 & $0.93,1.03$ & 1.01 & $0.96,1.06$ & 1.01 & $0.96,1.07$ & 0.89 & $0.79,1.00$ & 0.91 & $0.81,1.02$ & 0.93 & $0.82,1.04$ & 1.00 & $0.94,1.06$ & 1.03 & $0.97,1.09$ & 1.03 & $0.97,1.09$ \\
\hline Poultry for processed red meat & 0.90 & $0.85,0.95$ & 0.99 & $0.93,1.04$ & 0.99 & $0.93,1.04$ & 0.85 & $0.74,0.96$ & 0.97 & $0.85,1 \cdot 11$ & 0.99 & $0.86,1.14$ & 0.91 & $0.86,0.97$ & 0.99 & $0.93,1.06$ & 0.99 & $0.92,1.05$ \\
\hline Fish for red meat & 0.92 & $0.88,0.95$ & 0.96 & $0.92,1.00$ & 0.96 & $0.93,1.00$ & 0.83 & $0.75,0.90$ & 0.89 & $0.82,0.98$ & 0.90 & $0.82,0.99$ & 0.94 & $0.90,0.98$ & 0.97 & $0.93,1.02$ & 0.98 & $0.94,1.02$ \\
\hline Fish for unprocessed red meat & 0.95 & $0.91,0.99$ & 0.96 & $0.92,1.01$ & 0.97 & $0.93,1.02$ & 0.84 & $0.76,0.93$ & 0.87 & $0.79,0.97$ & 0.88 & $0.80,0.98$ & 0.98 & $0.93,1.02$ & 0.99 & $0.94,1.04$ & 0.99 & $0.95,1.04$ \\
\hline Fish for processed red meat & 0.87 & $0.83,0.91$ & 0.95 & $0.90,0.99$ & 0.95 & $0.90,1.00$ & 0.80 & $0.71,0.90$ & 0.93 & $0.82,1.06$ & 0.94 & $0.83,1.07$ & 0.89 & $0.85,0.94$ & 0.95 & $0.90,1.00$ & 0.95 & $0.90,1.00$ \\
\hline Lean fish for red meat & 0.99 & $0.94,1.05$ & 1.01 & $0.95,1.07$ & 1.00 & $0.95,1.07$ & 0.97 & $0.86,1.10$ & 1.00 & $0.89,1.14$ & 1.00 & $0.88,1.14$ & 0.99 & $0.93,1.06$ & 1.00 & $0.94,1.07$ & 1.00 & $0.94,1.07$ \\
\hline Fatty fish for red meat & 0.83 & $0.77,0.89$ & 0.90 & $0.83,0.96$ & 0.91 & $0.85,0.98$ & 0.66 & $0.56,0.78$ & 0.76 & $0.64,0.89$ & 0.78 & $0.66,0.91$ & 0.88 & $0.81,0.95$ & 0.94 & $0.87,1.02$ & 0.95 & $0.88,1.03$ \\
\hline Fish for poultry & 0.97 & $0.91,1.03$ & 0.96 & $0.90,1.02$ & 0.96 & $0.90,1.02$ & 0.95 & $0.83,1.09$ & 0.96 & $0.84,1.09$ & 0.95 & $0.83,1.09$ & 0.98 & $0.91,1.05$ & 0.96 & $0.90,1.03$ & 0.96 & $0.90,1.03$ \\
\hline Lean fish for poultry & 1.05 & $0.97,1.14$ & 1.01 & $0.94,1.09$ & 1.00 & $0.93,1.08$ & $1 \cdot 12$ & $0.95,1.33$ & 1.08 & $0.92,1.27$ & 1.06 & $0.90,1.24$ & 1.03 & $0.94,1 \cdot 12$ & 0.99 & $0.91,1.07$ & 0.99 & $0.91,1.07$ \\
\hline Fatty fish for poultry & 0.88 & $0.80,0.96$ & 0.90 & $0.82,0.98$ & 0.91 & $0.83,0.99$ & 0.76 & $0.63,0.93$ & 0.81 & $0.67,0.98$ & 0.82 & $0.68,0.99$ & 0.91 & $0.83,1.01$ & 0.92 & $0.84,1.02$ & 0.94 & $0.85,1.03$ \\
\hline \multicolumn{19}{|l|}{ Substitutions within main food groups } \\
\hline Unprocessed for processed red meat & 0.92 & $0.88,0.96$ & 0.98 & $0.94,1.03$ & 0.98 & $0.93,1.02$ & 0.95 & $0.84,1.08$ & 1.07 & $0.93,1.22$ & 1.07 & $0.93,1.22$ & 0.91 & $0.87,0.96$ & 0.96 & $0.92,1.01$ & 0.96 & $0.91,1.01$ \\
\hline Lean for fatty unprocessed red meat & 0.93 & $0.86,1.01$ & 0.98 & $0.91,1.07$ & 0.98 & $0.90,1.07$ & 0.94 & $0.76,1 \cdot 15$ & 0.99 & $0.81,1.21$ & 1.00 & $0.82,1.22$ & 0.93 & $0.85,1.02$ & 0.98 & $0.90,1.07$ & 0.97 & $0.89,1.07$ \\
\hline Fatty for lean fish & 0.83 & $0.75,0.93$ & 0.89 & $0.80,0.99$ & 0.91 & $0.82,1.01$ & 0.68 & $0.54,0.85$ & 0.75 & $0.60,0.94$ & 0.77 & $0.62,0.97$ & 0.88 & $0.78,1.00$ & 0.94 & $0.83,1.06$ & 0.95 & $0.84,1.07$ \\
\hline
\end{tabular}


(a)

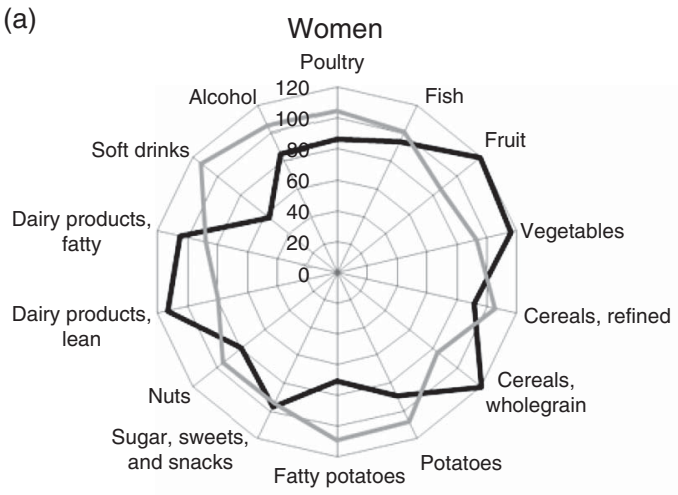

(b)

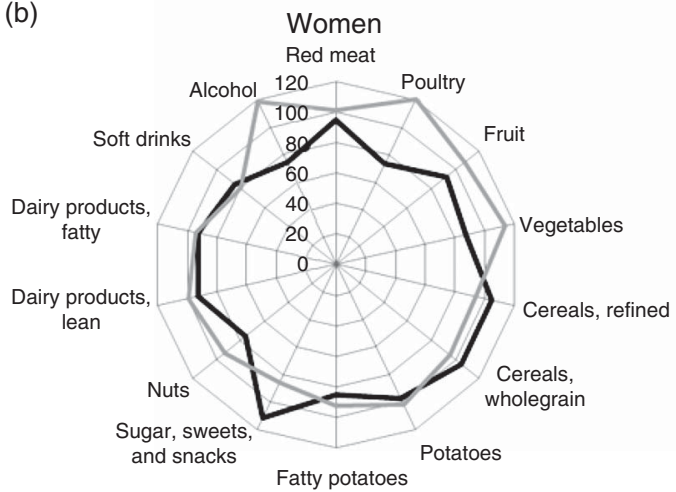

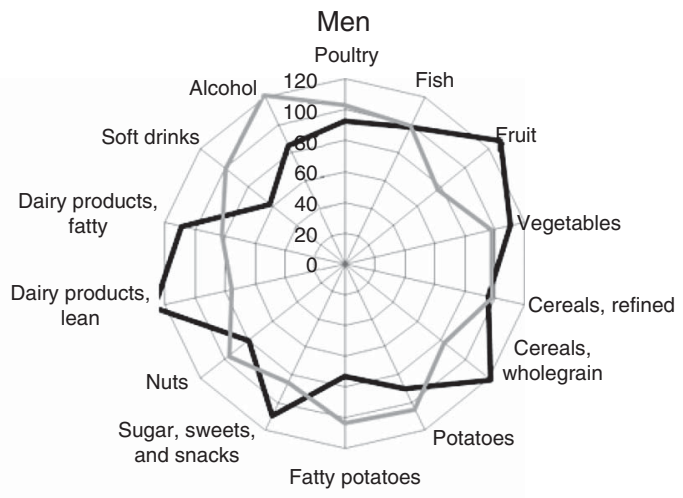

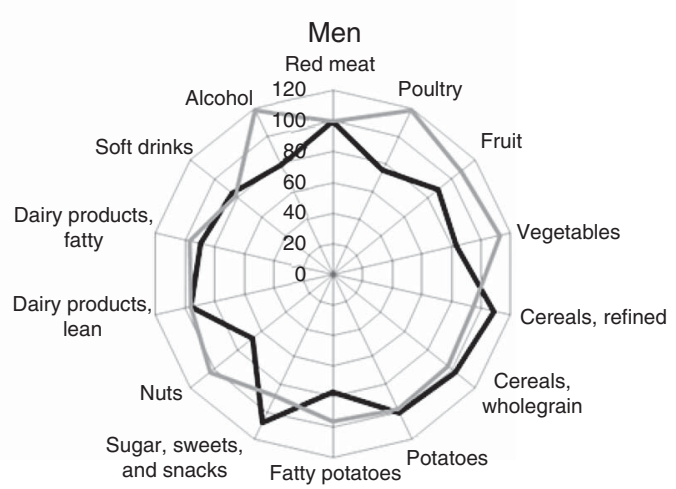

Fig. 1. Radar charts illustrating the percentage-wise differences in intake of fourteen groups of foods and beverages among women and men with the lowest and highest intakes of red meat (a) and fish (b); $100 \%$ is equivalent to the median intake of a particular food or beverage item among all participants of similar sex. First quintile; —_ fifth quintile.

of energy from the different foods. Overall, the results were similar, and only the results from substitutions of equal weight are presented.

Owing to the inclusion criteria, participants of higher socio-economic status were slightly over-represented in the study $\operatorname{cohort}^{(9)}$, but the investigated associations are believed to be homogeneous across socio-economic groups. The follow-up of participants in the study was nearly complete, which limits the concern for selection bias. However, we cannot exclude selection bias if death from other causes with a disease aetiology similar to that of MI is associated with the probability of developing MI. Study participants who died of other atherosclerotic diseases such as stroke most likely had a higher risk of MI than participants in general due to similarities in the underlying aetiology of MI and other atherosclerotic diseases. Thus, underestimation of the associations cannot be excluded.

The dietary information was obtained from self-administered FFQ, which inherently give rise to random measurement error. This generally leads to underestimation of the true association and to loss of statistical power. The variation in the dietary data was sufficient to investigate substitutions of amounts of meat and fish translatable into serving sizes easy for the general population to comply with. The comprehensive information on intake of subtypes of meat and fish also enabled us to perform a detailed examination of subgroup-specific associations. Although FFQ may reflect the habitual eating pattern and we aimed to assess the long-term association between different food choices and development of MI, repeated measures would have been preferred over a single baseline measure. Multiple measures can correct some measurement error, and in addition dietary intake might have changed during the relatively long follow-up. The use of validated and complete registries for follow-up in addition to the confirmation and validation of each case of MI minimises the risk of misclassification of the outcome. Finally, the diagnoses were established independently of the FFQ, and information bias is thus unlikely to have affected our results.

The detailed information on potential confounders in the study limits the risk of residual confounding, but confounding from unmeasured risk factors of MI cannot be excluded. Adjustment for known MI risk factors in our models weakened the risk estimates. This indicates confounding from lifestyle habits, health status and reproductive factors. Adjustment for other foods and food groups further attenuated our results, although only slightly. Confounding from other foods is expected, as meat and fish choices are most often followed by different accompaniments, which are part of an underlying dietary pattern that might be differentially associated with MI. Some of these differences in dietary patterns are evident from the radar charts in Fig. 1. With adjustment for other dietary factors, the food substitution per se was investigated, but the results could not be easily applied to a meal context. The results from analyses on meat and fish substitutions without adjustment for diet should be interpreted in the context of the underlying dietary patterns. 
Two previous studies on meat consumption and MI have investigated substitutions of meat with a specified replacement food. In a large follow-up study of US female nurses, Bernstein et $a l^{(3)}$ found that replacing one serving per day of red meat with poultry or fish was associated with a statistically significant 19-24\% lower risk of CHD. Our finding of an inverse association for replacement of red meat with fatty fish among women is in agreement with this finding. In a community-based follow-up study of US women and men, Haring et $a l^{(19)}$ found no association with CHD when red meat was replaced with poultry or fish and seafood or when processed meat was replaced with unprocessed red meat. The findings for poultry and processed meat replaced with unprocessed meat are in agreement with the observed results in this study. Our findings suggest that fatty fish is a better alternative to red meat and poultry than is lean fish. This could be explained by the higher content in fatty fish of $n-3$ PUFA with their presumed beneficial effects on $\mathrm{CHD}^{(20)}$. Thus, from a health perspective, a higher intake of fatty fish in place of red meat, poultry and lean fish is beneficial, but the potential sustainability challenges with an overall increased fish consumption should also be considered ${ }^{(21)}$. Even though we only found statistically significant protective associations among women, a similar pattern was observed among men. The sex difference in the underlying risk of MI may partly explain the weak associations among men ${ }^{(22)}$, as men have a higher baseline risk of MI than women and the measures of association are expressed relatively.

In conclusion, replacing red meat, poultry or lean fish with fatty fish was associated with a lower risk of MI. Thus, this study suggests that fatty fish is a favourable alternative to meat and lean fish items. Studies on individual dietary changes over time and subsequent risk of MI would add additional scientific value to elucidate the role of red meat, poultry and fish in MI development.

\section{Acknowledgements}

The authors thank the Danish Cancer Society and the staff at the Diet, Cancer and Health study for the collection and administration of data.

This work is part of the project 'Diet and prevention of ischemic heart disease: a translational approach' (DIPI, www. dipi.dk), which is supported by the Danish Council for Strategic Research (contract 0603-00488B). One-third of the fellowship was granted by the Graduate School of Health, Aarhus University. The primary data collection was funded by the Danish Cancer Society. The funding agencies had no role in the design, analysis or writing of this article.

The authors' contributions are as follows: A. M. L. W., K. O. and M. U. J. contributed to the study design; A. T. and K. O. collected the data; A. M. L. W. performed the statistical analyses; A. M. L. W., M. D. H., A. T., E. B. R., E. B. S., K. O. and M. U. J. were involved in the interpretation of the data and critical revision of the manuscript; A. M. L. W. wrote the manuscript. A. M. L. W. had primary responsibility for the final content. All the authors read and approved the final version of the manuscript.

The authors declare no conflicts of interest.

\section{References}

1. Dalen JE \& Devries S (2014) Diets to prevent coronary heart disease 1957-2013: what have we learned? Am J Med 127, 364-369.

2. Astrup A, Dyerberg J, Elwood P, et al. (2011) The role of reducing intakes of saturated fat in the prevention of cardiovascular disease: where does the evidence stand in 2010? Am J Clin Nutr 93, 684-688.

3. Bernstein AM, Sun Q, Hu FB, et al. (2010) Major dietary protein sources and risk of coronary heart disease in women. Circulation 122, 876-883.

4. van der A DL, Peeters PH, Grobbee DE, et al. (2005) Dietary haem iron and coronary heart disease in women. Eur Heart $J$ 26, 257-262.

5. Lee DH, Folsom AR \& Jacobs DR Jr (2005) Iron, zinc, and alcohol consumption and mortality from cardiovascular diseases: the Iowa Women's Health Study. Am J Clin Nutr 81, 787-791.

6. Micha R, Wallace SK \& Mozaffarian D (2010) Red and processed meat consumption and risk of incident coronary heart disease, stroke, and diabetes mellitus: a systematic review and meta-analysis. Circulation 121, 2271-2283.

7. Jakobsen MU, Dethlefsen C, Joensen AM, et al. (2010) Intake of carbohydrates compared with intake of saturated fatty acids and risk of myocardial infarction: importance of the glycemic index. Am J Clin Nutr 91, 1764-1768.

8. Li D, Siriamornpun S, Wahlqvist ML, et al. (2005) Lean meat and heart health. Asia Pac J Clin Nutr 14, 113-119.

9. Tjonneland A, Olsen A, Boll K, et al. (2007) Study design, exposure variables, and socioeconomic determinants of participation in Diet, Cancer and Health: a population-based prospective cohort study of 57,053 men and women in Denmark. Scand J Public Health 35, 432-441.

10. Overvad K, Tiønneland A, Haraldsdottir J, et al. (1991) Development of a semiquantitative food frequency questionnaire to assess food, energy and nutrient intake in Denmark. Int J Epidemiol 20, 900-905.

11. Tjønneland A, Overvad K, Haraldsdottir J, et al. (1991) Validation of a semiquantitative food frequency questionnaire developed in Denmark. Int J Epidemiol 20, 906-912.

12. Lauritsen J (1999) FoodCalc. http://www.ibt.ku.dk/jesper/ foodcalc/ (accessed 14 April 2015).

13. Haraldsdottir J, Tjonneland A \& Overvad K (1994) Validity of individual portion size estimates in a food frequency questionnaire. Int J Epidemiol 23, 786-796.

14. Tjonneland A, Haraldsdottir J, Overvad K, et al. (1992) Influence of individually estimated portion size data on the validity of a semiquantitative food frequency questionnaire. Int J Epidemiol 21, 770-777.

15. Luepker RV, Apple FS, Christenson RH, et al. (2003) Case definitions for acute coronary heart disease in epidemiology and clinical research studies: a statement from the AHA Council on Epidemiology and Prevention; AHA Statistics Committee; World Heart Federation Council on Epidemiology and Prevention; the European Society of Cardiology Working Group on Epidemiology and Prevention; Centers for Disease Control and Prevention; and the National Heart, Lung, and Blood Institute. Circulation 108, 2543-2549.

16. Joensen AM, Jensen MK, Overvad K, et al. (2009) Predictive values of acute coronary syndrome discharge diagnoses differed in the Danish National Patient Registry. J Clin Epidemiol 62, 188-194.

17. Willett W \& Stampfer MJ (1986) Total energy intake: implications for epidemiologic analyses. Am J Epidemiol 124, $17-27$. 
18. Willett WC, Howe GR \& Kushi LH (1997) Adjustment for total energy intake in epidemiologic studies. Am J Clin Nutr $\mathbf{6 5}$, 1220S-1228S ; discussion 1229S-1231S.

19. Haring B, Gronroos N, Nettleton JA, et al. (2014) Dietary protein intake and coronary heart disease in a large community based cohort: results from the Atherosclerosis Risk in Communities (ARIC) Study. PLOS ONE 9, e109552.
20. De Caterina R (2011) n-3 Fatty acids in cardiovascular disease. $N$ Engl J Med 364, 2439-2450.

21. Li D \& Hu X (2009) Fish and its multiple human health effects in times of threat to sustainability and affordability: are there alternatives? Asia Pac J Clin Nutr 18, 553-563.

22. Mosca L, Barrett-Connor E \& Wenger NK (2011) Sex/gender differences in cardiovascular disease prevention: what a difference a decade makes. Circulation 124, 2145-2154. 